\author{
Military Technical College \\ Kobry Elkobbah, \\ Cairo, Egypt
}

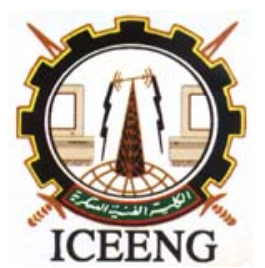

\title{
SYNTHESIS AND ANALYSIS OF NONLINEAR PREDICTIVE CONTROL FOR DIFFERENT PLANT REPRESENTATIONS
}

\author{
Ahmed $^{*}$ M. Youssef, Y. $^{*}$ Z. Elhalwagy
}

\begin{abstract}
:
A ducted fan has been constructed at California Institute of Technology, in order to provide an experimental test-bed for research and development of different control techniques for Uninhabited Combat Aerial Vehicles (UCAVs). It has many of the characteristics of existing flight control systems and its identification experiments have shown that its model is correct near hover and successful control designs also support this conclusion. This paper presents a comparison between the application of one of the nonlinear Model Based Predictive Control techniques (MBPC), which is called Non-Linear Quadratic Gaussian Predictive Control (NLQGPC), to two different representations of the ducted fan hover model. The paper also provides an insight into the strengths and weakness of each algorithm and into the types of problems, which limit the applicability of those algorithms.
\end{abstract}

Key words: Model Based Predictive Control (MBPC), Non-linear Quadratic Gaussian Predictive Control, State-Dependent, Linearization Around Trajectory (LAT), Flight simulation, Caltech ducted Fan, Uninhabited Combat Aerial Vehicles (UCAVs).

\section{INTRODUCTION}

A distinguishing feature of aerospace applications is the large envelope of operation regimes in which the process is usually highly nonlinear and has different characteristics from one operating condition to another. The process of designing flight control systems has been carried out traditionally by using linear systems analysis and design tools due to the availability of analytic solutions. However, in order to achieve tactical advantages, modern fighter aircraft strive towards performing maneuvers outside the region where the dynamics of flight are linear, and the need for nonlinear tools arises.

* Egyptian Armed Force

The high performance requirements of aerospace applications have always attracted the attention of researchers developing new advanced control algorithms. That is why in this 
paper, we investigate MBPC as a new framework for nonlinear flight control design. Because of the computational burden associated with the earlier MBPC techniques, applications of such techniques were prohibited for aerospace applications. This computational burden was resulted from utilizing the quadratic programming methods in solving the minimization problems numerically. In this paper, explicit optimal control laws are used, which can be solved analytically, results in removing the need for quadratic programming, thus can deal with the relatively fast dynamics systems.

This paper describes an attempt to apply MBPC to the design of a flight control system and presents a comparison between the application of one of the nonlinear MBPC techniques, which is called Non-Linear Quadratic Gaussian Predictive Control (NLQGPC), to two different representations of the ducted fan hover model. The first representation has a statedependent model structure, while the second one is the model linearized around trajectory. The comparison provides us with an insight into the strengths and weakness of each algorithm and into the types of problems, which limit the applicability of those algorithms.

The paper is organized as follows: In Section 2, the configuration of the ducted fan is described and its dynamics is discussed. Section 3 gives a description of the two algorithms of NLQGPC using the two different model representations. Nonlinear simulations of the closedloop system with the obtained controllers are presented in Section 4. In Section 5, a comparison between the two algorithms and a discussion of the types of problems, which limit the applicability of these algorithms are carried out. Finally, this paper concludes with a brief summary in Section 6.

\section{CALTECH DUCTED FAN MODEL}

The Caltech Ducted Fan is a small flight control experiment designed for research and development of nonlinear flight guidance and control techniques for UCAVs. It is a scaled model of the longitudinal axis of a flight vehicle [1]. Test results validate that the dynamics replicate qualities of actual flight vehicles, i.e. its dynamics are representative of either a Vertical/Short Take-Off and Landing (V/STOL) aircraft such as a Harrier in hover mode, or a thrust vectored aircraft such as F-18 HARV and X-31 in forward flight [2].

As shown in Figure 1, the fan is composed of a variable speed electric motor, which drives a four-blade propeller. The motor and the propeller assembly are bolted inside a wooden duct which has two flaps attached at the end. These flaps allow the thrust to be vectored from side to side and even reversed. The ducted fan engine is mounted on a rotating arm, which limits motion to three degrees of freedom: one rotational and two translational, approximately on the surface of a sphere defined by the arm. With this geometry, the ducted fan is completely controllable with just the vectored thrust. While this experimental system is considerably simpler than a real vectored thrust aircraft, it has many of the basic characteristics of more complicated flight control systems [1].

The full description of the construction of the Caltech ducted fan and the derivation of its full nonlinear model are detailed in [3]. In this paper, the planar model of the fan in the hover flight mode that ignores the stand dynamics is utilized. The equations of motion for this planar may be written as: 


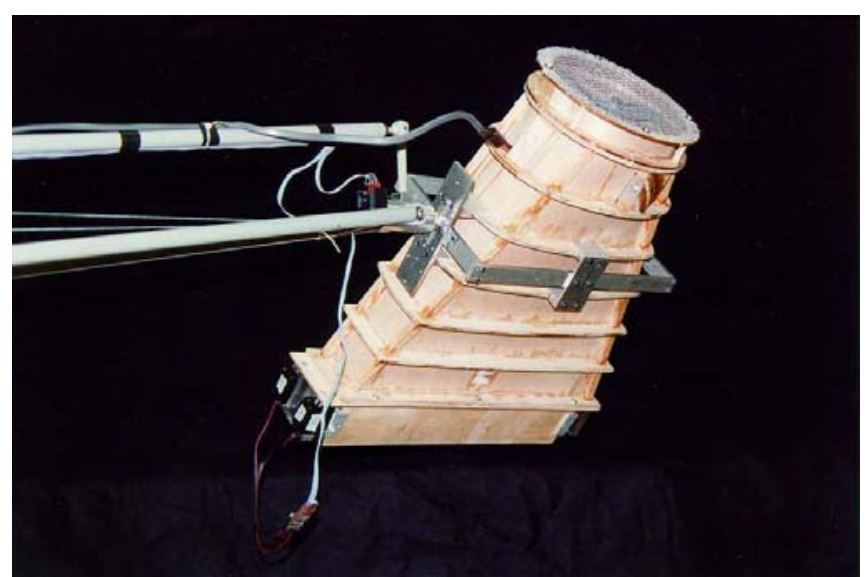

Figure 1: Caltech ducted fan

$$
\begin{aligned}
& m \underline{\ddot{x}}=f_{1} \cos \theta-f_{2} \sin \theta-c_{d, \underline{x}}(\theta, \underline{\dot{x}}) \\
& m \ddot{y}=f_{1} \sin \theta+f_{2} \cos \theta-m g-c_{d, y}(\theta, \dot{y}) \\
& J \ddot{\theta}=r f_{1}
\end{aligned}
$$

where $(\underline{x}, y)$ denote the position of the center of mass of the fan, while $\theta$ is the rotation of the fan about the boom axis. Figure 2 shows the simple planar model of the ducted fan.

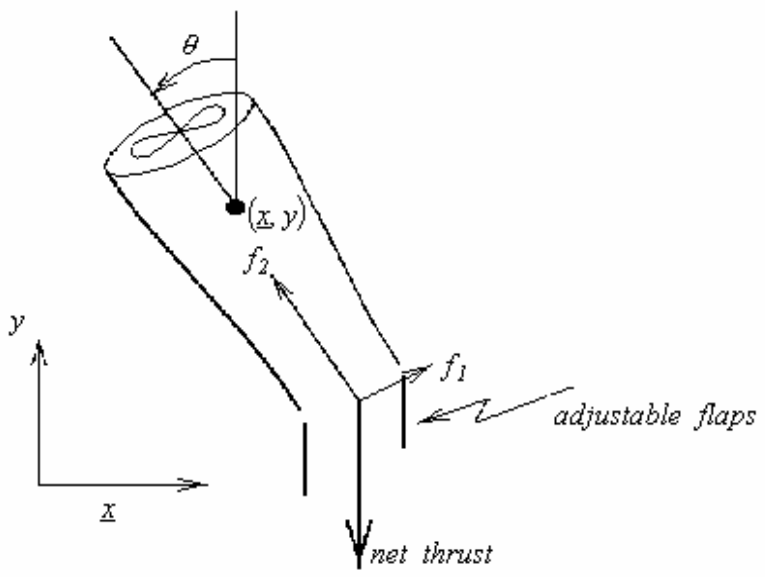

Figure 2: Planar ducted fan model

We assume that there are two forces acting on the fan: $f_{1}$ is perpendicular to the axis of the fan acting at a distance $r$, while $f_{2}$ is parallel to the axis of the fan. Let $m, J$, and $g$ be the mass of the fan, the moment of inertia, and the gravitational constant respectively. Redefine the inputs so that the origin is an equilibrium point of the system with zero input. Letting $v_{1}=f_{1}$ and $v_{2}$ $=f_{2}-m g$ and modeling the drag terms $\left(c_{d, x}, c_{d, y}\right)$ as viscous friction with $d$ being the friction coefficient, the equations become: 


$$
\begin{aligned}
& m \ddot{x}=-m g \sin \theta-d \dot{\dot{x}}+v_{1} \cos \theta-v_{2} \sin \theta \\
& m \ddot{y}=m g(\cos \theta-1)-d \dot{y}+v_{1} \sin \theta+v_{2} \cos \theta \\
& J \ddot{\theta}=r v_{1}
\end{aligned}
$$

These equations are referred to as the planar ducted fan equations. Using standard rigid body mechanics, the nonlinear state space model for the ducted fan can be easily constructed, which is represented by the following general equation:

$$
\begin{aligned}
& \dot{\xi}=f(\xi(t), v(t)) \\
& y=g(\xi(t))
\end{aligned}
$$

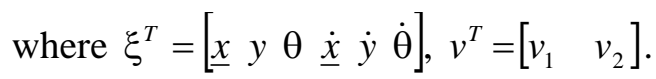

The numerical values for the parameters of this model are given in [2].

\section{NONLINEAR QUADRATIC GAUSSIAN PREDICTIVE CONTROL}

The NLQGPC is an extension of the LQG approach to nonlinear predictive control. This section describes the algorithms of applying the NLQGPC to the two different model representations: the state-dependent model, and the model linearized around trajectory.

\subsection{State-dependent Model Algorithm}

Equation (3) can be transformed into an alternative representation, with a linear-like structure having State-Dependent Coefficients (SDC) in the following form:

$$
\begin{aligned}
& \dot{\xi}=A(\xi(t)) \xi(t)+B(\xi(t)) v(t) \\
& y=\bar{C} \xi(t)
\end{aligned}
$$

where 


$$
\begin{aligned}
& A(\xi(t))=\left[\begin{array}{cccccc}
0 & 0 & 0 & 1 & 0 & 0 \\
0 & 0 & 0 & 0 & 1 & 0 \\
0 & 0 & 0 & 0 & 0 & 1 \\
0 & 0 & a_{43} & a_{44} & 0 & 0 \\
0 & 0 & a_{53} & 0 & a_{55} & 0 \\
0 & 0 & 0 & 0 & 0 & 0
\end{array}\right], B(\xi(t))=\left[\begin{array}{cc}
0 & 0 \\
0 & 0 \\
0 & 0 \\
b_{41} & b_{42} \\
b_{51} & b_{52} \\
b_{61} & 0
\end{array}\right], \\
& \bar{C}=\left[\begin{array}{llllll}
1 & 1 & 1 & 0 & 0 & 0
\end{array}\right]
\end{aligned}
$$

The coefficients of matrices $A(\xi(t))$ and $B(\xi(t))$ are given in Table 1 . System (4) is then discretized with sampling period $T_{s}$ and the integral action is incorporated as follows:

$$
\begin{aligned}
& \underbrace{\left[\begin{array}{c}
\xi(n+1) \\
v(n)
\end{array}\right]}_{x_{n+1}}=\underbrace{\left[\begin{array}{cc}
A(\xi(n)) & B(\xi(n)) \\
0 & I
\end{array}\right]}_{A_{n}} \underbrace{\left[\begin{array}{c}
\xi(n) \\
v(n-1)
\end{array}\right]}_{x_{n}}+\underbrace{\left[\begin{array}{c}
B(\xi(n)) \\
I
\end{array}\right]}_{B_{n}} \underbrace{u(n)}_{u_{n}} \\
& \underbrace{y(n)}_{y_{n}}=\underbrace{\left[\begin{array}{ll}
\bar{C} & 0
\end{array}\right]}_{C} \underbrace{\left[\begin{array}{c}
\xi(n) \\
v(n-1)
\end{array}\right]}_{x_{n}}
\end{aligned}
$$

The subscript $n$ denotes discrete time where $t=n T_{s}$, and $u_{n}=v_{n}-v_{n-1}$ is the control increment.

At each iteration, the predictions of the future state and control are used, instead of the future state and control which are not available, to recalculate the state-dependent state-space matrices within the algorithm's prediction horizon $N$. To simplify notation, the final matrices are denoted as $A_{n}, B_{n}, C$. Thus by computing a prediction of the future trajectory, the nonlinear system response is approximated by a known time-varying linear system. From these future state-space matrices, we calculate the following matrices as follows:

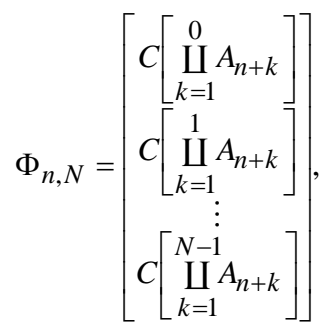




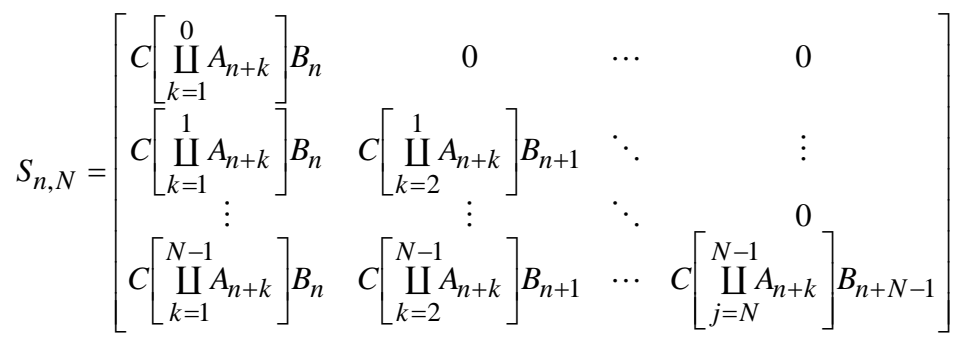

where

$$
\left[\begin{array}{l}
\bigsqcup_{k=l}^{n} A_{k}
\end{array}\right]=\left\{\begin{array}{cc}
A_{n} A_{n-1} \cdots A_{l} & \text { if } l \leq n \\
I & \text { if } l>n
\end{array}\right.
$$

We assume that after a long prediction horizon $N$, the state-space model matrices will be constant i.e. become time-invariant system. Therefore it is possible to obtain a solution for the Difference Riccati Equation (DRE) at that time $n+N$ and to then use it as a steady-state solution for solving the DRE backwards to $n=1$. Finally the optimal control law is calculated as follows:

$$
\begin{aligned}
& U_{n, N-1}=-\left(\Lambda_{U}+S_{n, N}{ }^{T} \Lambda_{E} S_{n, N}+\beta_{n}^{T} \tilde{H}_{n+1} \beta_{n}\right)^{-1} . \\
& {\left[\left(S_{n, N}{ }^{T} \Lambda_{E} \Phi_{n, N} A_{n}+\beta_{n}^{T} \tilde{H}_{n+1}^{1} A_{n}\right) x_{n}+\left(\beta_{n}^{T} \tilde{H}_{n+1}^{2} \Theta_{n, N}-S_{n, N}{ }^{T} \Lambda_{E}\right) R_{n+1, N}\right]}
\end{aligned}
$$

where $\Lambda_{E}^{i} \geq 0, i=1 \ldots N$ and $\Lambda_{U}^{j}>0, j=1 \ldots N-1$ are weighting matrices and $N \geq 1$ is an integer number. $R_{n+1, N}$ is a vector containing current and future values of setpoint $r_{n}$. $\beta_{n}$ is constructed from the matrix $B(\xi)$, given in Equation (5), as follows:

$$
\beta_{n}{ }^{T}=\left[\begin{array}{llll}
B^{T} & 0 & \cdots & 0
\end{array}\right]
$$

$\Theta_{n, N}$ denotes the transition matrix for the following reference signal model:

$$
\begin{aligned}
& X_{n+1}^{R}=\Theta_{n, N} X_{n}^{R} \\
& R_{n+1, N}=X_{n}^{R}
\end{aligned}
$$

where $\tilde{H}_{n+1}^{1}$ and $\tilde{H}_{n+1}^{2}$ are the solutions of the coupled Riccati equations. See [4] for a detailed derivation of this algorithm. The optimal solution (11) is obtained from minimizing the following infinite performance index: 


$$
\begin{aligned}
& J_{n}=\lim _{T \rightarrow \infty}\left\{\frac{1}{T+1} \sum_{k=n}^{n+T} J_{k}\right\} \\
& J_{k}=\sum_{j=1}^{N}\left\{\left(r_{k+j}-y_{k+j}\right)^{T} \Lambda_{E}^{j}\left(r_{k+j}-y_{k+j}\right)\right\}+ \\
& +\sum_{j=0}^{N-1}\left\{u_{k+j}{ }^{T} \Lambda_{U}^{j} u_{k+j}\right\}
\end{aligned}
$$

As any MBPC technique, at each iteration, only the first element of the calculated control vector is applied to the plant, but unlike other MBPCs, the remaining part of the control vector is used to predict future states (and consequently system matrices) by employing an appropriate numerical integration method. This idea is borrowed from Kouvaritakis et al. [5].

\subsection{Linearized Around Trajectory Model Algorithm}

An approach to nonlinear predictive control, utilizing the optimal control trajectory, calculated in the previous time instant of the control algorithm, was employed by Kouvaritakis et al. [5]. An extension of the previous optimal trajectory to the current time instant was referred by these authors as the "tail". In this approach, the system was linearized around this trajectory and then the linearized time-varying system was employed to obtain the optimal control, which is calculated as a perturbation from the "tail” trajectory. In Lee et al [6] a similar methodology, employing linearization at points of the seed trajectory was introduced, using a discrete time model representation of the system.

This is one of the steps involved in the underlying control algorithm, where at each iteration, the predictions of the future state and control are used, instead of the future state and control which are not available, to recalculate the trajectory dependent matrices by discretizing the model (3) with sampling period $T_{s}$ then linearizing it around a particular operating point $\left(\bar{\xi}_{n}, \bar{v}_{n}\right)$ on the predicted trajectory, and finally the integral action is incorporated as in Equations (6,7). The resulting system becomes in the form:

$$
\begin{aligned}
& x_{n+1}=\bar{A}_{n} x_{n}+\bar{B}_{n} \Delta u_{n} \\
& w_{n}=C x_{n}
\end{aligned}
$$

where

$$
x_{n}=\left[\begin{array}{c}
\xi_{n}-\bar{\xi}_{n} \\
u_{n-1}
\end{array}\right], \quad \begin{aligned}
u_{n-1} & =v_{n-1}-\bar{v}_{n-1} \\
\Delta u_{n} & =u_{n}-u_{n-1} \\
w_{n} & =y_{n}-\bar{y}_{n}
\end{aligned}
$$


To simplify notation, denote the matrices from equation (15) as $A_{n}=\bar{A}_{n}$, and $B_{n}=\bar{B}_{n}$. From these trajectory dependent matrices, we calculate the matrices $\Phi_{n, N}$ and $S_{n, N}$ as given in equations $(8,9)$. The cost function is given by:

$$
\begin{aligned}
& J_{n}=\lim _{T \rightarrow \infty}\left\{\frac{1}{T+1} \sum_{k=n}^{n+T} J_{k}\right\} \\
& J_{k}=\sum_{j=1}^{N}\left\{\left(r_{k+j}-w_{k+j}\right)^{T} \Lambda_{E}^{j}\left(r_{k+j}-w_{k+j}\right)\right\}+ \\
& +\sum_{j=0}^{N-1}\left\{\Delta u_{k+j}{ }^{T} \Lambda_{U}^{j} \Delta u_{k+j}\right\}
\end{aligned}
$$

which is minimized and the optimal solution is obtained as:

$$
\begin{aligned}
& \Delta U_{n, N-1}=-\left(\Lambda_{U}+S_{n, N}{ }^{T} \Lambda_{E} S_{n, N}+\beta_{n}^{T} \tilde{H}_{n+1}^{1} \beta_{n}\right)^{-1} . \\
& {\left[\left(S_{n, N}{ }^{T} \Lambda_{E} \Phi_{n, N} A_{n}+\beta_{n}^{T} \tilde{H}_{n+1}^{1} A_{n}\right) x_{n}+\left(\beta_{n}^{T} \tilde{H}_{n+1}^{2} \Theta_{n, N}-S_{n, N}{ }^{T} \Lambda_{E}\right) R_{n+1, N}\right]}
\end{aligned}
$$

where $R_{n+1, N}$ is the future setpoint vector for the model LAT after taking into account that the predicted trajectory differs from the actual one according to equation (16).

After calculating $\Delta U_{n, N-1}, U_{n, N-1}$ and consequently $V_{n, N-1}$ are calculated. The first element $v_{n}$ of $V_{n, N-1}$ is applied to the plant, while the remaining part of $V_{n, N-1}$ is used to predict future states (and consequently outputs) in the next iteration, as mentioned before. See [7] for a detailed derivation of this algorithm.

\section{SIMULATION RESULTS}

Comparison between the two controllers is performed in terms of the rise time/fall time, the settling time, the percent overshoot/undershoot, and the control effort. The setpoints for all the monitoring variables $(\underline{x}, y, \theta)$ are set to zero. The following tuning parameters are kept constant for the underlying controllers: the prediction horizon $N=6$, the sampling time $T_{s}=$ $250 \mathrm{~ms}$, the number of iterations of the Difference Riccati Equation (DRE) is set to 20, and

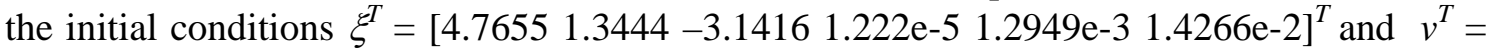
$[5.1251 \mathrm{e}-6-43.414]^{T}$. Asymptotic stability is ensured by augmenting the controllers with the satisficing approach [8], where the candidate Control Lyapunov Function (CLF) is given as:

$$
V(\eta)=\eta^{T} \Psi \eta
$$


$\eta$ is the state of the augmented plant-reference model after the integral action is incorporated, and the positive definite matrix $\Psi$ is chosen as follows:

$$
\Psi=\left[\begin{array}{cccc}
\mu & 0 & \cdots & 0 \\
0 & 0 & & \vdots \\
\vdots & & \ddots & \vdots \\
0 & \cdots & \cdots & 0
\end{array}\right]
$$

and $\mu=\operatorname{diag}\{3,7,10\}$.

For the state-dependent model algorithm, the weighting matrices are set to $\Lambda_{E}=\operatorname{diag}\{0.75$, $100,20\}$, and $\Lambda_{U}=\operatorname{diag}\{2,0.01\}$, whilst for the LAT model algorithm they have the following values $\Lambda_{E}=\operatorname{diag}\{3,7,45\}$, and $\Lambda_{U}=\operatorname{diag}\{0.8330 .005\}$.

The closed-loop responses for the NLQGPC implementations based upon the two approaches are plotted in Figures (3-7), where the solid line responses relate to the SDC model form approach.

Figure 3 displays the responses of the x-position of the ducted fan, where it can be seen that the NLQGPC-SDC exhibits the same fall time $t_{f}$, and almost the same percent undershoot (\% U.S.), but with shorter settling time $t_{s}$ compared to the performance of the NLQGPC-LAT, as listed in Table 2. The responses of the z-position of the ducted fan are shown in Figure 4, where the NLQGPC-SDC exhibits much faster fall time and quicker settling time than the NLQGPC-LAT, but displays more than 20 times higher value of percent undershoot. The behaviour of the z-position for the NLQGPC-LAT is non-smooth at $0.75 \mathrm{~s}$, due to the coupling between states, which increases its fall time. Figure 5 shows $\theta$ responses, where for the NLQGPC-LAT, it displays double the rise time $t_{r}$ compared to the performance of the NLQGPC-SDC, but exhibits almost no percent overshoot (\% O.S.), and shorter settling time. For the NLQGPC-SDC, the shorter rise time results in more oscillatory response with a maximum percent overshoot of $21.535 \%$.

Figures 6 and 7 show the trajectories of the two control inputs $v_{1}$ and $v_{2}$, where the control

effort of $v_{1}$ is almost the same for both controllers, whilst the control effort of $v_{2}$ is much lower in case of the NLQGPC-LAT than the NLQGPC-SDC.

\section{COMPARISONS AND DISCUSSIONS}

\subsection{Tracking Performance}

The tracking performance represents the most visible measure. Although from the simulation results, both algorithms are capable of achieving high degree of tracking performance and prove their capabilities to be applied to fast dynamic systems, the LAT approach can produce slightly better performance NLQGPC controllers than the SDC approach. 


\subsection{Applicability to Different Plants}

In case of the state-dependent model algorithm, some difficulties arise when trying to transform a general nonlinear model (3) to the SDC form. First, the lack of a systematic procedure for selecting, among the infinite possibilities, a single parameterization for matrices $A(\xi(t))$ and $B(\xi(t))$, which achieves acceptable performance. Second, this method requires the pair $(A(\xi(t)), B(\xi(t)))$ be point-wise controllable in the linear sense for all the state $\xi$, and due to the non-uniqueness of $A(\xi(t))$ and $B(\xi(t))$, different choices of $A(\xi(t))$ and $B(\xi(t))$ may yield different controllability matrices and thus different point-wise controllability characteristics. Third, different $A(\xi(t))$ choices yield different locations of the system eigenvalues, and consequently different "dynamic structure” of the physical system. Fourth, numerical problems can arise when one state difference equation is parameterized in such a way that is nearly the same as another, i.e., a multiple of the other.

All of these difficulties must be taken into consideration if the plant models are affine nonlinear systems, as in system (2). For inherently nonlinear systems in which the input variables may appear in non-affine forms, as in aircraft models, chemical reactions, $\mathrm{PH}$ neutralisation and distillation columns, obtaining SDC forms are extremely difficult. But in case of the LAT model algorithm, the nonlinear plant, whatever its representation, is linearized around the tail and this linearized time-varying system is employed to obtain the optimal control. Thus this algorithm can be applied to any nonlinear system.

\section{CONCLUSIONS}

This paper described an attempt to apply a nonlinear predictive control technique to a real fast dynamic flight control system. It proposed two algorithms using two different plant representations for the same nonlinear predictive control technique. The first representation has a state-dependent model structure, while the second one is the model linearized around trajectory. The two algorithms proved their capabilities of both achieving high degree of tracking performance and dealing with fast system dynamics.

Discussion on the strengths and weaknesses of each of these algorithms has concluded that the requirement on the plant model to be described in a SDC form is considered to be a limitation to design problem for particular application. In other words, these methods can be only applied to a special class of affine nonlinear systems. Thus for inherently nonlinear systems, it is recommended to use local linearization of the nonlinear model along the calculated trajectory. 


\section{REFERENCES}

[1] Kantner M., Bodenheimer B., Bendotti P., and Murray R.M., An Experimental Comparison of Controllers for a Vectored Thrust, Ducted Fan Engine. In Proceedings of the American Control Conference, Pages 1956-1961, Seattle, 1995.

[2] Murray R.M., Modeling of the Caltech Ducted Fan, class notes for cds 111. Technical report, California Institute of Technology, Control and Dynamical Systems 107-81, Pasadena, CA91125, 1998.

[3] Choi H., Sturdza P., and Murray R. M., Design and Construction of a Small Ducted Fan Engine for Nonlinear Control Experiments, In Proceedings of the American Control Conference, Pages 2618-2622, Maryland, 1994.

[4] Grimble M. J., Ordys A. W., Non-linear Predictive Control for Manufacturing and Robotic Applications, IEEE Conference on Methods and Models in Automation and Robotics, Poland, August 2001.

[5] Kouvaritakis B., Cannon M., and Rossiter J. A., Non-linear model based predictive control, Int. J. Control, vol. 72, no. 10, pp. 919-928, 1999.

[6] Lee Y.I., Kouvaritakis B., and Cannon M., Constrained receding horizon predictive control for nonlinear systems, Automatica, vol.38, no.12, pp. 2093-2102, 2002.

[7] Youssef A. M., Grimble M. J., Ordys A. W., Dutka A., and Anderson D., Robust Nonlinear Predictive Flight Control, In Proceedings of the European Control Conference, Cambridge, 2003.

[8] Youssef A. M., Stable Nonlinear Predictive Control for Fast Constrained Systems, Proceedings of the 5th International Conference on Electrical Engineering (ICEENG), May, 2006.

Table 1: Performance indices

\begin{tabular}{|l|l|}
\hline $\begin{array}{l}\text { Coefficients of } \\
\text { matrix } A\end{array}$ & $\begin{array}{l}\text { Coefficients of } \\
\text { matrix } B\end{array}$ \\
\hline$a_{43}=-g \sin (\theta) / \theta$ & $b_{41}=\cos (\theta) / m$ \\
$a_{44}=-d / m$ & $b_{42}=-\sin (\theta) / m$ \\
$a_{53}=g(\cos (\theta)-1) /$ & $b_{51}=\sin (\theta) / m$ \\
\hline
\end{tabular}

Table 2: Performance indices

\begin{tabular}{|c|c|c|c|}
\hline \multirow{2}{*}{$\begin{array}{l}\frac{0}{0} \\
. \overrightarrow{7} \\
\frac{\pi}{7} \\
>\end{array}$} & \multirow[b]{2}{*}{$\begin{array}{l}\text { Criteri } \\
\mathrm{a}\end{array}$} & \multicolumn{2}{|l|}{ Controllers } \\
\hline & & $\begin{array}{l}\text { NLQGPC } \\
(\mathrm{SDC})\end{array}$ & $\begin{array}{l}\text { NLQGPC } \\
(\mathrm{LAT})\end{array}$ \\
\hline \multirow{3}{*}{$\mathrm{X}$} & $t_{f}[\mathrm{~s}]$ & 1.25 & 1.25 \\
\hline & $t_{s}[\mathrm{~s}]$ & 2 & 2.5 \\
\hline & \% U.S. & 0.111 & 0.063 \\
\hline \multirow{3}{*}{$\mathrm{Z}$} & $t_{f}[\mathrm{~s}]$ & 0.25 & 1.25 \\
\hline & $t_{s}[\mathrm{~s}]$ & 1 & 1.5 \\
\hline & \% U.S. & 30.53 & 0.056 \\
\hline \multirow{3}{*}{$\theta$} & $t_{r}[\mathrm{~s}]$ & 0.5 & 1 \\
\hline & $t_{s}[\mathrm{~s}]$ & 2.25 & 1.75 \\
\hline & \% O.S. & 21.53 & 0.005 \\
\hline
\end{tabular}




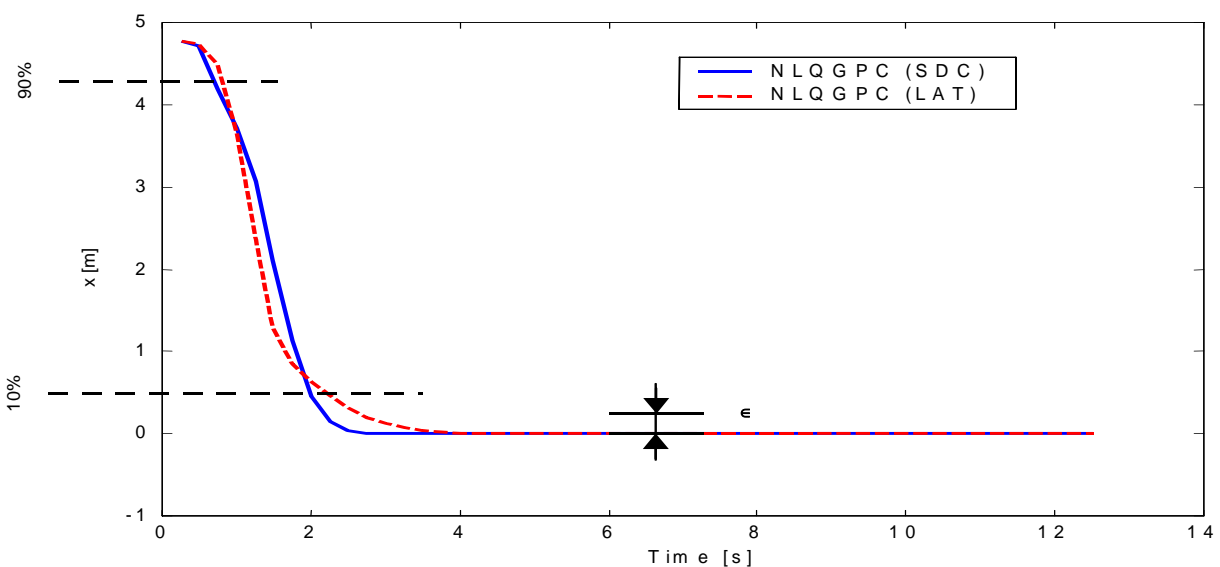

Figure 3: Responses of the x-position

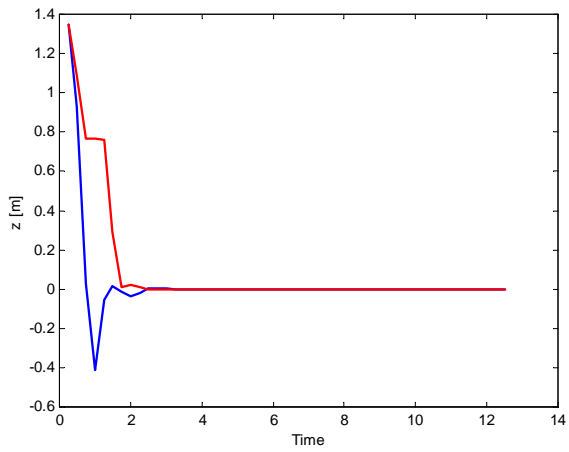

Figure 4: Responses of the z-position

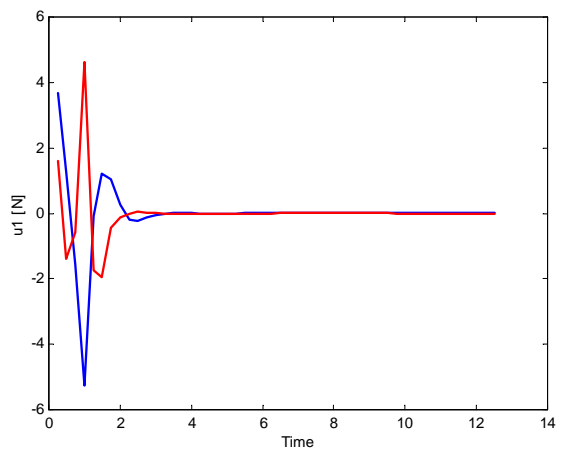

Figure 6: Trajectories of $v_{1}$

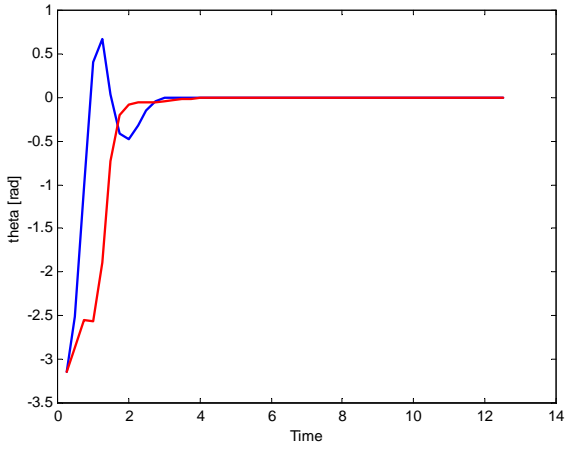

Figure 5: The responses of $\theta$

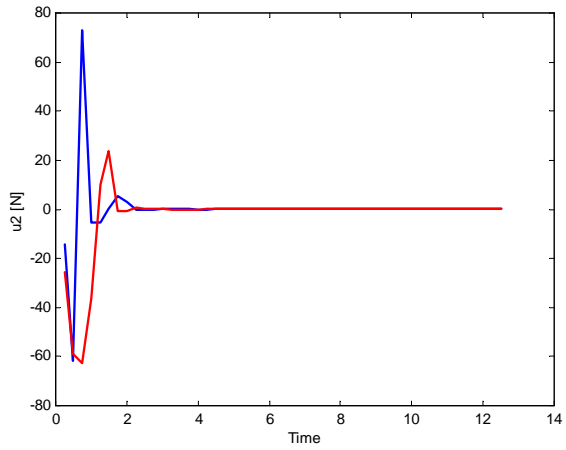

Figure 7: Trajectories of $v_{2}$ 